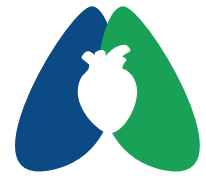

ASSOBRAFIR

C I Ê N C I A

\section{As intervenções fisioterapêuticas motoras e seus efeitos em pacientes de unidade de terapia intensiva adulto: uma revisão integrativa da literatura}

\author{
Physical therapy interventions and their effects in intensive \\ care patients: integrative literature review
}

\author{
Zulamar Aguiar Cargnin ${ }^{1}$ (1), Norma Beatriz Dias Rangel ${ }^{2}$ (b) \\ 1 Universidade Vale do Rio Verde (Unincor), Três Corações, MG, Brasil \\ ${ }^{2}$ Secretaria de Estado da Saúde, Florianópolis, SC, Brasil
}

Como citar: Cargnin ZA; Rangel NBD. As intervenções fisioterapêuticas motoras e seus efeitos em pacientes de unidade de terapia intensiva adulto: uma revisão integrativa da literatura. ASSOBRAFIR Ciênc. 2021;12:e42492. https://doi.org/10.47066/2177-9333.AC.2020.0024

\begin{abstract}
Resumo
Introdução: A intervenção fisioterapêutica motora pode prevenir ou diminuir a fraqueza muscular de pacientes em tratamento intensivo. Objetivo: descrever as intervenções fisioterapêuticas motoras e seus efeitos nos desfechos clínicos de pacientes adultos em UTI. Métodos: revisão integrativa de literatura. As buscas aconteceram nas bases Scielo, PubMed, Scopus e Embase com artigos em inglês, espanhol e português de 2012 a 2021. O caminho metodológico foi conduzido com o auxílio do software Atlas Ti. Resultados: Foram incluídas 21 publicações. Estas, imergiram três categorias temáticas: "mobilização precoce", "cicloergômetro" e "estimulação elétrica neuromuscular". A avaliação pela escala PEDro mostrou o predomínio de pesquisas de média qualidade Conclusões: 0 interesse dos últimos anos concentrou-se na mobilização precoce, estimulação elétrica neuromuscular e exercícios em cicloergômetro. Devido à qualidade metodológica média dos estudos e aos desfechos heterogêneos há, ainda, evidências insuficientes para guiar a prática clínica.

Palavras-chave: Unidades de Terapia Intensiva; Modalidades de Fisioterapia; Cuidados Críticos; Mobilização Precoce; Estimulação Elétrica.

Abstract

Background: Physical therapy interventions can prevent or decrease muscle weakness in intensive care patients. Aim: describe motor physical therapy interventions and their effects on the clinical outcomes of adult ICU patients. Methods: integrative literature review. The searches took place in the Scielo, PubMed, Scopus and Embase databases with articles in English, Spanish and Portuguese from 2012 to 2021. The methodological path was conducted with the aid of the Atlas Ti software. Results: 21 publications were included. Three thematic categories were immersed: "early mobilization", "cycle ergometer" and "neuromuscular electrical stimulation". The evaluation using the PEDro scale showed the predominance of medium quality research. Conclusion: the interest of recent years has been concentrated on early mobilization, neuromuscular electrical stimulation and application of the cycle ergometer. Due to the average methodological quality of the studies and the heterogeneous outcomes, there is still insufficient evidence to guide clinical practice.
\end{abstract}

Keywords: Intensive care Units; Physiotherapy Modalities; Critical Care; Early Mobilization; Electric Stimulation. 


\section{INTRODUÇÃO}

O paciente crítico que permanece tempo prolongado em Unidade de Terapia Intensiva (UTI) está sujeito a complicações provocadas pela baixa mobilidade 1 . As disfunções ocorrem precocemente e podem perdurar por até 5 anos após a doença original ${ }^{2}$. Portanto, melhorar os resultados a longo prazo para esses pacientes, torna-se clinicamente relevante ${ }^{3}$.

A Fraqueza Adquirida na UTI (FMA-UTI) é um agravo que tem uma incidência de, pelo menos, $25 \%$ a $33 \%$ e a baixa mobilidade apresenta-se como um dos fatores de risco ${ }^{4}$. Nesse sentido, a mobilização precoce pode ajudar a reduzir as incapacidades funcionais dos pacientes com fraqueza muscular ${ }^{5}$.

A intervenção fisioterapêutica motora é uma importante estratégia de prevenção da fraqueza muscular, evita excesso de sedação e delirium e potencializa a recuperação do paciente ${ }^{2}$. Programas precoces podem beneficiar pacientes com insuficiência respiratória aguda, prevenir ou atenuar a fraqueza neuromuscular adquirida na UTI e são seguros e viáveis ${ }^{3}$. Diminuem também o tempo de permanência na UTI bem como no hospital'1.

A mobilização precoce é uma estratégia importante, no entanto, seus efeitos clínicos não estão bem estabelecidos ${ }^{1}$. Adicionalmente, apesar de sua aplicação, os pacientes ainda desenvolvem fraqueza muscular que pode ser atribuída a baixa intensidade dos exercícios no leito e a limitação pela presença do tubo orotraqueal, sedação e estado mental ${ }^{6}$. A reabilitação precoce, com a estimulação elétrica neuromuscular e o exercício em cicloergômetro, tem sido, também, investigada sem uma conclusão clara sobre sua eficácia e seus efeitos ${ }^{6,7}$. Porém, ainda faltam mais pesquisas que determinem os efeitos das intervenções motoras nos desfechos de pacientes de cuidados críticos.

Nesse sentido, este estudo objetivou descrever as intervenções fisioterapêuticas motoras e seus efeitos nos desfechos clínicos de pacientes adultos de UTI.

\section{MÉTODOS}

Trata-se de uma revisão integrativa da literatura. Foram incluídos ensaios clínicos randomizados e quase randomizados. As buscas foram realizadas eletronicamente através da biblioteca digital Scientific Electronic Librare Online (Scielo) e bases de dados US National Library of Medicine (PubMed), Scopus e Excerpta Médica dataBASE (Embase). A seleção se deu com artigos publicados em inglês, português ou espanhol no período de 2012 a 2021, disponíveis na íntegra. Inseridas, também, publicações detectadas nas referências dos artigos selecionados.

As fases dessa revisão foram: determinação da pergunta norteadora, escolha das fontes de dados, eleição das palavras chave, definição dos critérios de inclusão e exclusão, busca e seleção da amostra, coleta de dados e armazenamento, avaliação dos artigos, análise e interpretação dos dados e apresentação das evidências encontradas. A pergunta norteadora foi: quais as intervenções fisioterapêuticas motoras e seus efeitos nos desfechos clínicos de pacientes adultos em UTI ? Para atingir os objetivos propostos, procedeu-se a inclusão do artigo pelo título; seguida pela apreciação dos resumos; e, por último, pela leitura mais atenta e cuidadosa na íntegra. A estratégia de busca está na Tabela 1.

Os critérios de inclusão foram: ensaios clínicos com população adulta sob cuidados críticos que descreviam a aplicação de intervenção motora, bem como os desfechos clínicos dos pacientes. Foram excluídas publicações que envolviam crianças, modelos animais, protocolos de ensaios clínicos e artigos de revisão.

Os estudos selecionados foram avaliados quanto à qualidade metodológica através da escala PEDro com onze itens, sendo que 10 itens são pontuados por um ponto (1) significando a presença do indicador de qualidade e zero (0) não continham a informação ou não cumpriam a condição do indicador de qualidade. É baseada na lista Delphi desenvolvida na Holanda e elaborada pela Physiotherapy Evidence Database (PEDro). Faz uma avaliação da validade interna dos estudos e comparações estatística e, desse modo, promove maior credibilidade aos resultados. A generalização dos resultados, a relevância clínica e a magnitude do efeito das intervenções não são avaliadas. Possui uma moderada confiabilidade entre examinadores.

Tabela 1. Estratégia de busca.

\begin{tabular}{cc}
\hline & ("rehabilitation") AND ("Critical care") AND \\
SCIELO & year_cluster: ("2012 OR "2013 OR “2015" OR \\
"2016" OR “2017" OR"2018" OR "2019" OR "2020" \\
OR "2021")
\end{tabular}

((“Early ambulation" OR "Physical therapy modalities" OR "rehabilitation")) AND ("Critical care")

Filters activated: Publication date from 2012/01/01 to 2021/04/30, Humans, Critical Trial, Full text.

\section{Estratégia PICO:}

PUBMED Patient/Problem/Medical condition: Physical therapy in Intensive care units

Intervention: Physical therapy modalities, Electric stimulation

Outcome: Rehabilitation.

"Physical therapy modalities" AND Intensive care units

\begin{tabular}{|c|c|}
\hline SCOPUS & $\begin{array}{l}\text { "Electric Stimulation" AND Intensive care units } \\
\text { Filters activated: } 2012 \text { to } 2021\end{array}$ \\
\hline EMBASE & $\begin{array}{c}\text { ('early ambulation'/exp OR 'early ambulation' OR } \\
\text { 'physical therapy modalities'/exp OR 'physical } \\
\text { therapy modalities') AND ('critical care'/exp OR } \\
\text { 'critical care') AND [embase]/lim NOT ([embase]/ } \\
\text { lim AND [medline]/lim) AND ('clinical trial'/de OR } \\
\text { 'controlled study'/de OR 'randomized controlled } \\
\text { trial'/de) AND ‘article'/it AND ([adult]/lim OR [aged]/ } \\
\text { lim OR [middle aged]/lim OR [very elderly]/lim OR } \\
\text { [young adult]/lim) }\end{array}$ \\
\hline
\end{tabular}


Os estudos foram classificados em três categorias: baixa qualidade metodológica (1 a 4 pontos), média qualidade metodológica (5 a 7) e alta qualidade (8 a 10) $)^{8}$. Os artigos com escore $<5$ foram excluídos.

O estudo foi conduzido com o auxílio de software Atlas Ti que foi projetado em 1989 na Alemanha para auxiliar na análise de pesquisas qualitativas. Acelera o processo inferencial através do gerenciamento dos arquivos e da codificação e permite armazenar uma grande quantidade de dados em vários formatos. Além disso, proporciona mais rigor científico e metodológico ${ }^{9}$. Foi utilizada a versão 8 do Atlas ti adquirida através de uma licença para estudante por seis meses. São gerados códigos e próprio software faz a contagem da frequência de códigos e de outras funcionalidades. Os relatórios gerados (Outputs) permitem as análises inferenciais com frações de texto importantes para conduzir e dar base a discussão.

A exploração dosmanuscritos aconteceu com a introdução dos artigos selecionados no Atlas.ti como documentos (documents). Posteriormente, foram selecionados trechos significativos destacados e marcados no ponto original dos documentos originando citações (quotations). Selecionadas livremente com comentários (comments) ou associadas a códigos (codes) para a análise descritiva ou atribuição de um sentido aos segmentos selecionados. Os códigos primários foram: tamanho da amostra, alocação, autores, conclusão, critérios de inclusão e exclusão, desfechos primários, discussão, grupo intervenção e grupo controle, justificativa, limitações, local, medidas realizadas, objetivo, protocolo de tratamento, resultados, sugestão para futuros estudos, técnica utilizada e delineamento do estudo.

Através do gerenciador de códigos do software, explorou-se o código "técnica utilizada" e a partir dele foram criados códigos secundários: "Mobilização precoce", "Cicloergômetro" e "Estimulação elétrica neuromuscular". Daí imergiu um grupo de códigos (Code groups) chamado "Categorias norteadoras" que organizaram os dados, facilitaram a sua interpretação e serviram de pré categoria de análise (Figura 1). Os resultados foram analisados qualitativamente com comparações entre grupos e descrição dos principais desfechos.

\section{RESULTADOS}

A busca identificou 1633 artigos acrescidos de 16 publicações de outras fontes e resultaram em 21 estudos ( $n=2131$ pacientes). O fluxograma da busca encontra-se na Figura 2. As publicações concentraram-se mais nos anos de 2016, 2018 e 2019, representando 61,9\% do total de estudos selecionados. O tamanho da amostra apresentou uma variação de 16 a 308 participantes adultos, de ambos os gêneros, com média de idade de 33 a 77 anos, submetidos à Ventilação Mecânica Invasiva (VMI). Eventos adversos relatados pelos estudos foram poucos ou raros e sem consequências graves.

As técnicas fisioterapêuticas aplicadas foram heterogêneas e com protocolos variados, diferentes medidas de resultados

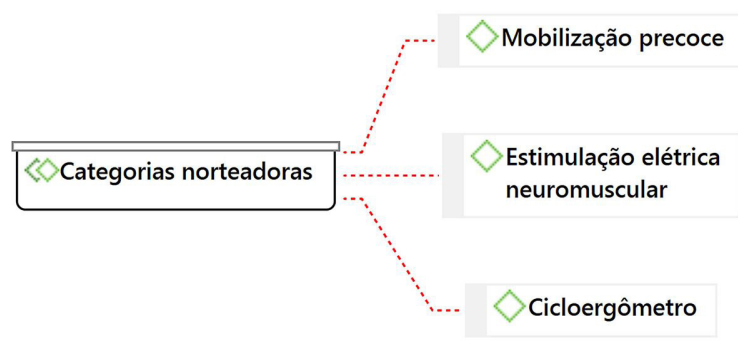

Figura 1. Categorias norteadoras extraídas do software Atlas ti.

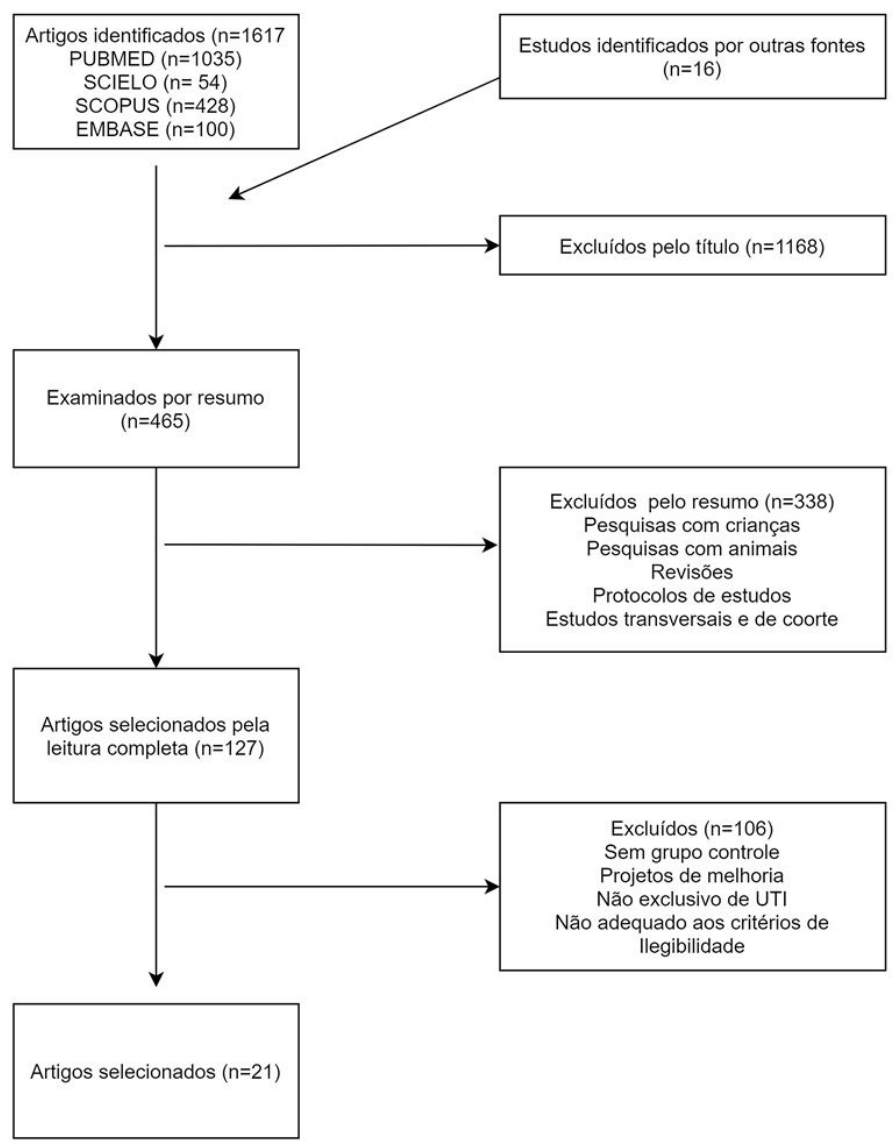

Figura 2. Fluxograma dos estudos selecionados.

e, geralmente, não envolviam acompanhamentos pós UTI. Os desfechos e principais resultados encontram-se na Tabela 2. A relação dos artigos, número de pacientes, estratégia utilizada e qualidade metodológica são fornecidas no Tabela 3.

A avaliação da validade interna e qualidade metodológica pela escala PEDro mostrou o predomínio de 19 pesquisas de média qualidade (escore 5 a 7) e duas de alta qualidade (escore 8 a 10). A pontuação média foi 6,10 (Desvio Padrão, 1,04) de um total de 10 pontos. Com exceção de dois artigos 6,12, os escores foram extraídos diretamente da base de dados PEDro (Tabela 3). Os critérios menos atendidos foram os itens: 3 (alocação oculta dos sujeitos), 5 (sujeitos cegos), 6 (terapeuta cego), 7 (acompanhamento adequado) e 8 (análise por intenção de tratar). 
Tabela 2. Características dos artigos quanto aos desfechos e principais resultados.

\begin{tabular}{lc}
\hline \multicolumn{1}{c}{ Estudos } & Desfechos \\
\hline 1.Rodriguez et al.,2012 & Força muscular do bíceps e quadríceps \\
\hline a. Força muscular de MMSS e MMII (melhora no \\
processo de desmame)
\end{tabular}

2.Akar et al.,2015 11

b. Tempo de alta da UTI

c. Mediadores inflamatórios

\section{Resultados}

(grupo intervenção vs grupo controle)

Bíceps 4 (3-4) vs 3 (2-4); p = 0,005 e quadríceps 3 (3-4) vs 3 (2-3); $p=0,034$

a. Grupo1 - MMII 3 para 5, $p=0,014$ e MMSS de 4 para $5 p=0,038$; Grupo2 - MMII 4 para 5, $p=0,046$ e MMSS de 4 para 5, $p=0,046$

Grupo3 - MMII não houve melhora, MMSS de 4 para 5, $p=0,034$

b. Semelhante para os grupos, $p=0,240$

c. Diminuição do nível sérico de interleucina 6 no Grupo1 e interleucina 8 no Grupo1 e Grupo2

3. Yosef-

Brauner et al.,2015

a. Força muscular dos MMSS e MMII pelo MRC

b. Tempo de permanência na UTI

a. Espessura da camada muscular

4. Fischer et al.,2016 ${ }^{12}$

b. Força muscular dos MMSS e MMII pelo MRC

5. Morris et al.,2016 ${ }^{13}$

Tempo de permanência no hospital

a. Melhora no grupo intervenção; $p<0.05$

b. $13 \pm 4,6$ dias vs $18,11 \pm 3,1$ dias; $p=0,043$.

a. Sem efeito significativo nos dois grupos

b. O grupo intervenção recuperou a força muscular 4,5 vezes mais rápido que o grupo controle

10 dias (IIQ, 6 -17) vs 10 dias (IIQ, $7-16$ ). Diferença mediana, 0 (IC 95\%-1.5 - 3), p = 0,41)

Escores totais de CS-PFP-10

6. Moss et al.,2016

7. Schaller et al.,2016 ${ }^{14}$
Capacidade de realizar AVD pela teste de Desempenho Funcional Físico do CS-PFP-10 em 1, 3 e 6 meses nos três momentos ( $p=0,73,0,29$ e 0,43, respectivamente) e trajetória total do escore CS-PFP-10 $(p=0,71)$. a. Nível de mobilização por um olgaritmo de pontuação de mobilização ótima

\section{a. 2,2 vs $1,5, p<0,0001$}

b. Redução do tempo de UTI

c. Eventos adversos. b. 7 dias (DP 5-12) vs 10 dias (DP 6-15), $p=0,054$

c. 25 casos no $(2,8 \%)$ vs 10 casos $(0,8 \%)$ no controle.

a. Aumento (basal vs. final) tanto no grupo intervenção $(38,73 \pm 11,11$ vs. $47,18 \pm 8,75$; a. Força muscular periférica, $p<0,001)$ como no controle $(40,81 \pm 7,68$ vs. 45,00 $\pm 6,89 ; p<0,001$ ); a variação do aumento da força foi maior no grupo intervenção que no controle $(8,45 \pm 5,20)$ vs. $(4,18 \pm 2,63 ; p=0,005)$

\section{Machado et al.,2017}

\section{b. Tempo de UTI}

c. Tempo de ventilação mecânica

d. Tempo de internação hospitalar

$$
\begin{aligned}
& \text { b. } p=0,824 \\
& \text { c. } p=0,715
\end{aligned}
$$$$
d \cdot p=0,794
$$

\section{a. Capacidade funcional pelo TC6}

a. $123 \mathrm{~m}$ (IIQ 25-280) vs $100 \mathrm{~m}$ (IIQ 0-300), $p=0,542$

b. Independência funcional na alta hospitalar pelo MIF b. 98 (IIQ 66-119) vs 98 (IIQ 18-115), p=0,308. 48 (IIQ, 29-58) vs 51 (IIQ, 37-58) pontos, $\mathrm{p}=0,28$

a. Preservação da área transversa da fibra muscular $(12,4 \% \pm 22,5 \%$ no grupo intervenção vs $25,8 \% \pm 21,6 \%$ no controle; $p=0,005$ )

a. Área transversa da fibra muscular

11. Hickmann et al., $2018^{16}$

b. Regulação das vias de degradação /síntese de proteínas no choque séptico b. A ativação excessiva da autofagia devido ao choque séptico foi menor no grupo intervenção, sem ser suprimido. A intervenção não aumentou a inflamação e foi bem tolerada

\section{Medrinal et al.,2018 ${ }^{17}$}

13. Santos et al.,2018 18

14. Wright et al., $2018^{2}$
Débito cardíaco durante o exercício

Duração da ventilação mecânica
Somente aumentou no grupo do cicloergômetro com EENM, média de $1 \mathrm{~L} / \mathrm{min}(15 \%)$.

Terapia combinada $(5,7 \pm 1,1)$ e EENM $(9,0 \pm 7,0)$ vs grupo controle $(14,8 \pm 5,4)$.
$37(12,2)$ vs $37(11,3)$ com uma diferença ajustada em média-1,1 (IC 95\%-7,1 a 5,0).

p-valor- nível de significância $\mathrm{p}<0,05$; MMSS - membros superiores; MMII - membros inferiores; UTI - unidade de terapia intensiva; MRC - Medical Research Council; IIQ - intervalo interquatil; IC - intervalo de confiança; AVD - atividades de vida diária; CS-PFP-10 - Continuous-Scale Physical Functional Performance Test; DP - desvio padrão; TC6 - Teste de Caminhada de 6 minutos; MIF - Medida de Independência Funcional; EENM - estimulação elétrica neuromuscular; SF-36 - Outcomes Short-Form Health Survey; DEN - Distúrbio Eletrofisiológico Neuromuscular. 
Tabela 2. Continuação...

\begin{tabular}{|c|c|c|}
\hline \multirow{2}{*}{ Estudos } & \multirow{2}{*}{ Desfechos } & Resultados \\
\hline & & (grupo intervenção vs grupo controle) \\
\hline \multirow{3}{*}{ 15. Sarvati et al., $2018^{19}$} & a. Força muscular pelo MRC & a. $50(45-56)$ vs $48(45-54) ; p=0,555$ \\
\hline & b. Fraqueza antes da mobilização & b. $92 \%$ vs $80 \%, p=0,045$ \\
\hline & c. Recuperação muscular & c. $14(10-24)$ vs $10(5-15), p=0,004$ \\
\hline \multirow[b]{2}{*}{ 16. Santos et al.,20196 } & a. Parâmetros cardiovasculares & $\begin{array}{c}\text { a. Não houve diferença entre os grupos antes, } \\
\text { durante e após o protocolo. }\end{array}$ \\
\hline & b. Mecânica respiratória & $\begin{array}{l}\text { b. Discreta elevação da resistência do sistema } \\
\text { respiratório no grupo cicloergometria e uma } \\
\text { redução da mesma no grupo-controle }\end{array}$ \\
\hline \multirow{3}{*}{ 17. Silva et al., $2019^{20}$} & $\begin{array}{l}\text { a. Perda da espessura muscular do tibial anterior e } \\
\text { reto femoral }\end{array}$ & $\begin{array}{c}\text { a. }-1 \% \text { (IC 95\% - } 4 \text { a 3) e - 0,04 mm } \\
\text { (IC 95\% - 0,11 a 0,02), p=0,15 vs perda } \\
\text { de - 0,33 mm (- 14\%) e - 0,49 mm (- 21\%), p<0,0001 }\end{array}$ \\
\hline & b. DEN & b. $0 \%$ vs. $47 \%, p<0,0001$, razão de risco de 16 \\
\hline & c. Pico de força evocado & c. $(2,34 \mathrm{~kg} / \mathrm{f}, p<0,0001)$ vs $(-1,55 \mathrm{~kg} / \mathrm{f}, p<0,0001)$. \\
\hline \multirow{4}{*}{ 18. Chen et al., $2019^{21}$} & a. Força muscular do quadríceps pelo MRC & $\begin{array}{l}\text { a. Mediana, IIQ, de } 2(1-3,5) \text { para } 2(1-4) \text { pontos, } \\
p=0,034 \text { vs de } 1(1-2) \text { para } 1(1-2,5), p>0,99\end{array}$ \\
\hline & b. Circunferência da perna esquerda & $\begin{array}{c}\text { b. 39,7 (DP5,6 cm) para 38,8 (DP 5,1 cm), } p=0,38 \text { vs } \\
47,5(\mathrm{DP} 8,3 \mathrm{~cm} \text { ) para 44,6 (DP 5,7 cm), } p=0,004 .\end{array}$ \\
\hline & c. Espessura de dobra cutânea & $\begin{array}{c}\text { c. (IIQ, 10,5 [6,8-15,8] mm para 10,0 }[5,8-14] \mathrm{mm} \text {, } \\
p=0,034) \text { vs } 13,0[6-19] \text { para } 14,5[8,5-19,3] \mathrm{cm} \\
p=0,92)\end{array}$ \\
\hline & d. Escores do MIF & $\begin{array}{c}\text { d. de 29,0 (DP14,5) para 32,0 (DP18,6), } \\
p=0,12 \text { vs 25,6 (DP7,9) para 25,2(DP 14,5), p=0,65 }\end{array}$ \\
\hline \multirow{3}{*}{$\begin{array}{l}\text { 19. Nakamura et al., } \\
2019^{22}\end{array}$} & a. Volume do músculo reto femoral & a. Diminuiu para ambos os grupos $(p<0,001)$ \\
\hline & b. Perda de volume muscular & $\begin{array}{l}\text { b. 10,4\% (DP10,1\%)(IC 95\% 5,8-15,1) vs 17,7\% } \\
\text { (DP10,8\%) (IC 95\% 11,9-23,5), } p=0.04\end{array}$ \\
\hline & c. Índice de Barthel para subir escadas & c. 3,9 (DP 4,0) vs $1,5(\mathrm{DP} 1,5), p=0,04$ \\
\hline \multirow{5}{*}{$\begin{array}{l}\text { 20. Schujmann et al. } \\
2020^{23}\end{array}$} & a. Porcentagem de pacientes independentes & a. $96 \%$ vs $44 \%, p<0,001$ \\
\hline & b. Tempo gasto em atividade & b.7,55\% vs 4,18\%, p <0,001 \\
\hline & c. Dias de UTI. & c. $5(4-7)$ vs $8(5-12), p=0,003$ \\
\hline & d. Estado funcional na alta da UTI. & d. $97 \pm 5$ vs $76 \pm 20, p<0,001$ \\
\hline & $\begin{array}{l}\text { e. Independência funcional } 3 \text { meses após a alta } \\
\text { pelo índice de Bartel }\end{array}$ & e. $39(97,5 \%)$ vs $29(74,4 \%), p=0,003$ \\
\hline \multirow{3}{*}{ 21. Segers et al., $2021^{24}$} & $\begin{array}{l}\text { a. Diminuição da espessura muscular do } \\
\text { quadríceps. }\end{array}$ & a. $-6 \pm 16 \%$ vs $-12 \pm 15 \%, p=0,014$ \\
\hline & b. Força muscular & b. Foi comparável nos dois grupos. \\
\hline & $\begin{array}{c}\text { c. Marcadores morfológicos e moleculares do } \\
\text { quadríceps. }\end{array}$ & $\begin{array}{l}\text { c. Músculos estimulados: maiores miofibras e } \\
\text { maior expressão do gene MyHC-I. }\end{array}$ \\
\hline
\end{tabular}

p-valor- nível de significância p< 0,05; MMSS - membros superiores; MMII - membros inferiores; UTI - unidade de terapia intensiva; MRC - Medical Research Council; IIQ - intervalo interquatil; IC - intervalo de confiança; AVD - atividades de vida diária; CS-PFP-10 - Continuous-Scale Physical Functional Performance Test; DP - desvio padrão; TC6 - Teste de Caminhada de 6 minutos; MIF - Medida de Independência Funcional; EENM - estimulação elétrica neuromuscular; SF-36 - Outcomes Short-Form Health Survey; DEN - Distúrbio Eletrofisiológico Neuromuscular.

Tabela 3. Relação dos artigos, tamanho da amostra, técnica utilizada e escore PEDro.

\begin{tabular}{|c|c|c|c|c|}
\hline Estudo/ano/país & $\mathbf{N}^{\circ}$ pctes & Estratégia & Escore PEDro & $\begin{array}{c}\text { Qualidade } \\
\text { metodológica }\end{array}$ \\
\hline 1.Rodriguez et al./2012/ Argentina10 & 16 & $\begin{array}{c}\text { Estimulação elétrica } \\
\text { neuromuscular }\end{array}$ & $6 / 10$ & Média \\
\hline 2. Akar et al./2015/Turquia ${ }^{11}$ & 30 & $\begin{array}{c}\text { Estimulação elétrica } \\
\text { neuromuscular }\end{array}$ & $5 / 10$ & Média \\
\hline 3.Yosef-Brauner et al./2015/ Israel $^{4}$ & 18 & Mobilização precoce & $5 / 10$ & Média \\
\hline 4. Fischer et al./2016/ Austria ${ }^{12}$ & 54 & $\begin{array}{c}\text { Estimulação elétrica } \\
\text { neuromuscular }\end{array}$ & $7 / 10$ & Média \\
\hline 5. Morris et al./2016/USA ${ }^{13}$ & 247 & Mobilização precoce & $5 / 10$ & Média \\
\hline
\end{tabular}


Tabela 3. Continuação...

\begin{tabular}{|c|c|c|c|c|}
\hline Estudo/ano/país & $\mathbf{N}^{\circ}$ pctes & Estratégia & Escore PEDro & $\begin{array}{l}\text { Qualidade } \\
\text { metodológica }\end{array}$ \\
\hline 6. Moss et al./2016/USA ${ }^{3}$ & 120 & Mobilização precoce & $6 / 10$ & Média \\
\hline $\begin{array}{l}\text { 7. Schaller et al./2016/ Austria, } \\
\text { Alemanha e Estados Unidos }{ }^{14}\end{array}$ & 200 & Mobilização precoce & $5 / 10$ & Média \\
\hline 8.Machado et al./2017/Brasil ${ }^{1}$ & 38 & Cicloergômetro & $5 / 10$ & Média \\
\hline 9.Eggman et al./2018/Suiça ${ }^{5}$ & 115 & Mobilização precoce & $7 / 10$ & Média \\
\hline 10.Fossat et al./2018/França ${ }^{15}$ & 314 & $\begin{array}{l}\text { Cicloergômetro + } \\
\text { Estimulação elétrica } \\
\text { neuromuscular }\end{array}$ & $7 / 10$ & Média \\
\hline 11.Hickmann et al./ 2018/ Bélgica ${ }^{16}$ & 21 & Cicloergômetro & $6 / 10$ & Média \\
\hline 12. Medrinal et al./2018/França ${ }^{17}$ & 20 & $\begin{array}{l}\text { Cicloergômetro + } \\
\text { Estimulaçãa elétrica } \\
\text { neuromuscular }\end{array}$ & $5 / 10$ & Média \\
\hline 13. Santos et al./2018/Brasil ${ }^{18}$ & 48 & $\begin{array}{l}\text { Estimulação elétrica } \\
\text { neuromuscular }\end{array}$ & $6 / 10$ & Média \\
\hline 14.Wright et al./ 2018/ Reino Unido² & 308 & Mobilização precoce & $8 / 10$ & Alta \\
\hline 15. Sarvati et al./ 2018/França ${ }^{19}$ & 125 & Mobilização precoce & $7 / 10$ & Média \\
\hline 16.Santos et al./2019/Brasil ${ }^{6}$ & 30 & Cicloergômetro & $5 / 10$ & Média \\
\hline 17. Silva et al., $2019^{20}$ & 40 & $\begin{array}{l}\text { Estimulação elétrica } \\
\text { neuromuscular }\end{array}$ & $8 / 10$ & Alta \\
\hline 18. Chen et al./2019/Taiwan²1 & 37 & $\begin{array}{l}\text { Estimulação elétrica } \\
\text { neuromuscular }\end{array}$ & $7 / 10$ & Média \\
\hline 19.Nakamura et al./ 2019/Japão22 & 94 & $\begin{array}{l}\text { Estimulação elétrica } \\
\text { neuromuscular }\end{array}$ & $5 / 10$ & Média \\
\hline 20. Schujmann et al./2020/Brasil ${ }^{23}$ & 99 & Mobilização precoce & $6 / 10$ & Média \\
\hline 21. Segers et al./2021/Bélgica ${ }^{24}$ & 47 & $\begin{array}{l}\text { Estimulação elétrica } \\
\text { neuromuscular }\end{array}$ & $7 / 10$ & Média \\
\hline
\end{tabular}

\section{DISCUSSÃO}

Nesta revisão foram analisados 21 estudos com intervenções motoras obtidos em ensaios clínicos randomizados. As técnicas utilizadas, as medidas, os resultados obtidos foram heterogêneos. Na maioria das terapias, o paciente e a equipe assistente não puderam ser cegados, mas os avaliadores conseguiram fazer uma avaliação cega. Algumas amostras foram pequenas e comprometeram a validade interna dos estudos com estimativas pouco precisas. Somam-se a isso o fato que, em pacientes críticos, os resultados de uma intervenção podem ser influenciados por várias variáveis fisiológicas e terapêuticas comprometendo as análises e causando limitações ${ }^{25}$.

Quanto à qualidade metodológica dos estudos, a maioria foi de grau moderado na avaliação da escala PEDro. No entanto, é necessário ainda melhorar a qualidade metodológica das pesquisas nacionais e internacionais e implementar uma prática clínica baseada em evidências disponíveis e buscar tratamentos eficazes para os pacientes ${ }^{26}$.

\section{Mobilização precoce}

A FMA-UTI ainda não está totalmente elucidada, mas é provavelmente devida a uma combinação de atrofia muscular e processos inflamatórios com envolvimento dos músculos esqueléticos e dos nervos periféricos ${ }^{5,27}$. Pode levar a resultados negativos como falha no desmame, internação prolongada na UTI, aumento da mortalidade e pior estado funcional com incapacidade persistente em atividades de vida ${ }^{5}$.

A reabilitação de pacientes graves tem se concentrado na disfunção muscular periférica e menos na reabilitação muscular respiratória ${ }^{28}$. Nesse sentido, a mobilização precoce mostra-se promissora. No entanto, o nível de mobilização precoce ainda é baixo principalmente naqueles pacientes com disfunção orgânica e que precisam mais de ventilação mecânica ${ }^{29}$.

A mobilização precoce é definida como a intensificação e a aplicação de fisioterapia nos primeiros 2-5 dias da doença crítica desde que haja estabilidade 1 . É a atividade física suficiente para provocar efeitos fisiológicos agudos que melhoram a ventilação, perfusão central e periférica, circulação, metabolismo muscular e alerta. Previne a estase venosa e trombose venosa profunda ${ }^{30}$. Tem-se mostrado também segura e viável com poucos eventos adversos registrados ${ }^{31}$. O início da mobilização dentro de 48-72 horas de VMI pode ser ideal para melhorar os resultados clínicos ${ }^{32}$.

Os desfechos dos artigos na categoria mobilização precoce foram diversos com resultados conflitantes. 
Os estudos não deixaram claro o momento exato de início das intervenções e houve diferentes abordagens intervencionais. Alguns não melhoraram os resultados físicos nem o tempo de permanência hospitalar 2,3,5,13.

Os efeitos de duas terapias de reabilitação precoce não melhoraram o desempenho da função física a longo prazo $^{2,3}$. Seus autores sugerem que em estudos futuros, os pesquisadores devem considerar a determinação de características que identifiquem os pacientes que necessitam e poderiam se beneficiar de um programa intensivo, bem como estabelecer os componentes específicos mais benéficos para os pacientes ${ }^{3}$. Outra intervenção realizada com treinamento de resistência não melhorou a capacidade funcional ou a independência na alta hospitalar e acreditam que a intervenção de reabilitação mais eficaz para estimular as adaptações neuromusculares ainda precisa ser determinada ${ }^{5}$. O estudo de Morris et al. (2016) ${ }^{13}$ comparou uma terapia de reabilitação padronizada com cuidados habituais em pacientes com insuficiência respiratória aguda e detectou que a intervenção não diminuiu o tempo de internação hospitalar. Também, a estratégia de verticalização passiva do paciente numa mesa de inclinação associada a fisioterapia convencional não melhorou a força muscular na alta da UTI nem o tempo de ventilação e permanência no hospital, mas os dados do estudo sugerem uma recuperação mais rápida com a inclinação ${ }^{19}$.

Outras pesquisas incluídas foram mais promissoras com melhora da função motora do paciente durante a internação na UTI, seu tempo de permanência e a mobilidade funcional na alta hospitalar ${ }^{4,14}$. A utilização da mobilização precoce direcionada por objetivos usando um algoritmo de mobilização estrito combinado com a comunicação interprofissional facilitada, melhorou a mobilização do paciente, diminuiu o tempo de permanência na UTI e melhorou a mobilidade funcional na alta hospitalar ${ }^{14}$. A chave do sucesso pode ter sido a sua implementação rigorosa ${ }^{14}$. É possível que um protocolo de terapia intensiva possa facilitar o processo de recuperação inicial em pacientes que sofrem de FMA-UTI ${ }^{4}$.

Outro estudo que comparou um programa de mobilidade progressiva com cinco níveis de atividades, exercícios e componentes cognitivos, mostrou melhora no estado funcional, na independência e tempo de internação na UTI. Não houve, no entanto, benefícios na força muscular periférica e respiratória. A funcionalidade é multifatorial, integra funções, estruturas corporais, estado de saúde e os fatores contextuais. Dessa forma, a força pode ser recuperada ou preservada mesmo com déficits pontuais por meio de restruturação e adaptação dos sistemas corporais pela atividade. Esse fato pode explicar a melhora nos resultados funcionais após a alta e meses depois ${ }^{23}$

Os estudos apresentaram algumas limitações que diminuíram os impactos das intervenções como a aplicação do programa somente durante a semana, dificuldades de acompanhar os pacientes aos 6 meses que culminou com dados faltantes para os desfechos secundários, início da primeira sessão, em média, 8 dias após o início da ventilação mecânica e um tamanho de amostra relativamente pequeno comprometendo os resultados ${ }^{2-5,14}$. Um ponto forte foi a intervenção fisioterapêutica aplicada em 48 horas de admissão na UTI ${ }^{5,23}$.

Revisões sistemáticas mostram resultados promissores como a melhora no estado de mobilidade, força muscular e sobrevivência fora do hospital aos seis meses, menor permanência VMI, diminuição de tempo de internação hospitalar e maior qualidade de vida ${ }^{27,33}$. Ao contrário, outras revisões não detectaram efeito significativo no tempo de VMI, no número de casos de FMA-UTI e mortalidade, mas apresentaram melhora na mobilidade funcional e foram eficazes na prevenção da fraqueza ${ }^{34,35}$.

A diretriz desenvolvida com base em revisão sistemática afirma que a mobilização precoce é segura ${ }^{36}$. Os eventos adversos não são frequentes e graves, estão relacionados com alterações hemodinâmicas e/ou respiratórias, de baixa frequência e reversíveis com a interrupção da intervenção. A mobilização precoce deve ser o objetivo de toda a equipe que deve considerar a estabilidade hemodinâmica e respiratória do paciente ${ }^{36,37}$.

Projetos de melhoria de qualidade têm implementado a mobilização precoce com bons resultados, segurança e viabilidade da intervenção ${ }^{38,39}$. Esses programas podem levar a maior conscientização e uma transformação na cultura dentro de toda a UTI ${ }^{38}$.

Os benefícios da mobilização precoce e atitudes que tornam favorável à sua realização são conhecidas pelos profissionais. Entretanto, sua aplicação é um desafio, principalmente pela indisponibilidade de pessoal e de tempo, nível de sedação e delirium dos pacientes, risco de autolesão musculoesquelética e excesso de estresse no trabalho ${ }^{40}$. Podem ser citados também preocupações de segurança, atrasos no reconhecimento de pacientes elegíveis, má comunicação e coordenação interdisciplinar, equipamento e protocolos insuficientes ${ }^{41}$. As barreiras encontradas são potencialmente modificáveis ${ }^{40}$.

Um importante fator contribuinte para má adesão a mobilização precoce são as barreiras culturais. Podem ser transpostas por uma abordagem multidisciplinar com comunicação e dando ênfase a importância da reabilitação precoce. Outra alternativa seria a implementação de uma equipe de mobilidade que não aumenta custos adicionais na contabilização geral, diminuindo riscos de readmissão ou morte ${ }^{34}$.

A principal barreira na mobilização precoce ainda é a sedação, apesar de necessária, atrasa a recuperação funcional dos pacientes e limita a intervenção fisioterapêutica ${ }^{1}$. O excesso de sedação pode se minimizado com testes diários de despertar ${ }^{42}$. Um estudo australiano apontou que há necessidade de estratégias como diminuir a sedação e/ou aumentar a equipe para melhorar os resultados de mobilidade dos pacientes ${ }^{43}$. Outro estudo 
sugeriu a atuação de uma equipe de mobilidade dedicada, liderada por fisioterapeutas no ambiente da UTI para enfrentar os obstáculos identificados ${ }^{44}$

Dessa forma, são necessários ensaios clínicos randomizados com a aplicação da intervenção motora e avaliação dos desfechos clínicos com critérios bem estabelecidos. Seria recomendável focar naqueles pacientes com mais tempo de VMI e uma definição bem determinada do que é considerado precoce ${ }^{29}$.

\section{Exercício em cicloergômetro}

A utilização do cicloergômetro pode aumentar a capacidade e auto percepção funcional e a força do quadríceps ${ }^{45}$. Conforme determinado pelo nível de vigília, participação e esforço do paciente, o dispositivo permite que haja uma atividade progressiva entre o ciclo passivo, ativo-assistida e ciclagem ativa e com resistência. Em particular, essa intervenção pode ser mais adequada para pacientes em UTI que não toleram atividades fora da cama, como ficar em pé, transferir para a cadeira ou caminhar ${ }^{41}$.

Cinco ensaios clínicos eleitos nessa revisão avaliaram a intervenção com exercício em cicloergômetro associado ou não a Estimulação Elétrica Neuromuscular (EENM)1,6,15-17. Os diferentes protocolos estudados e tamanhos amostrais podem ter influenciado nos resultados. Foram relatados poucos eventos adversos respiratórios/hemodinâmicos, mas sem consequências graves. Os resultados sugerem que houve auxilio na recuperação da força muscular periférica de pacientes internados em UTI; porém, o uso do cicloergômetro com exercício passivo não alterou o tempo de VMI e de internação hospitalar, não melhorou a força muscular quando comparado à reabilitação precoce isoladamente com exercícios passivos, ativos, transferência para a beira do leito ou cadeira, ortostatismo e treino de marcha conforme evolução clínica do paciente ${ }^{1,15}$. Já o estudo detectou que a intervenção fisioterapêutica precoce com exercícios passivos/ativos e cicloergômetro passivo/ativo por trinta minutos duas vezes ao dia durante a primeira semana de choque séptico é segura, preserva a área transversal da fibra muscular, não aumenta a inflamação muscular e é bem tolerada ${ }^{16}$. Quando procurou-se demonstrar a resposta cardiorrespiratória fisiológica e do débito cardíaco ao exercício, seus autores demonstraram que nenhum músculo ou efeitos sistêmicos foram induzidos pelas técnicas passivas e apenas o exercício em cicloergômetro associado com a EENM aumentou o débito cardíaco e a resposta cardiorrespiratória fisiológica com indução do consumo muscular de oxigênio e aumento do metabolismo muscular ${ }^{17}$. Seus efeitos sobre as alterações da mecânica respiratória e parâmetros cardiovasculares não foram significativos e induz um baixo nível de trabalho muscular ${ }^{6}$.

O ciclismo não altera a mecânica respiratória, nem a hemodinâmica e não resulta em respostas fisiológicas agudas ou necessidade de qualquer terapia adicional ${ }^{41,46}$. No entanto, essas intervenções motoras não foram suficientemente estudadas em pacientes críticos para saber se devem ser incorporadas em programas de reabilitação padronizados ${ }^{15}$.

Outros aspectos devem ser considerados como o acesso ao pessoal de reabilitação a equipamentos especializados, a estabilidade fisiológica dos pacientes, a experiência e treinamento do profissional. Finalmente, o momento ideal para o seu início e as indicações para o seu uso ainda não são conhecidos ${ }^{41}$.

\section{Estimulação elétrica neuromuscular (EENM)}

A EENM caracteriza-se pela aplicação de uma corrente elétrica aos músculos, através de eletrodos colocados na pele, que ativa os ramos nervosos intramusculares e induz a contração muscular ${ }^{47}$. É frequentemente realizada para preservar ou melhorar a massa muscular, força e função, especialmente dos músculos comprometidos de pacientes imobilizados e naqueles incapazes de colaborar ${ }^{48}$.

Alguns estudos incluídos nessa revisão apresentaram potenciais efeitos positivos da EENM. O estudo com pacientes com exacerbação da Doença Obstrutiva Crônica mostrou que a EENM sozinha e associada com exercício ativo parece superior ao treinamento muscular ativo de extremidade isolado para prevenir e aumentar a força muscular dos membros inferiores; no entanto, as duas intervenções não diferiram na duração do desmame e no tempo de alta da UTI ${ }^{11}$. Houve uma diminuição significativa na frequência cardíaca no grupo de EENM sozinho que pode significar que a estimulação melhora a força muscular sem aumentar a carga cardíaca. Também mostrou uma redução da resposta inflamatória durante o período de ventilação mecânica nos pacientes que realizaram EENM associado a exercícios ativos e no grupo que recebeu apenas EENM que pode significar que não aumentou a resposta inflamatória ${ }^{11}$. Esta intervenção pode aumentar o recrutamento das fibras musculares, reduzir a atrofia devido a imobilidade e fornecer efeitos sistêmicos semelhantes ao do exercício ${ }^{21}$.

A EENM associada com exercícios ativos foi bem tolerada e resultou em menor tempo de VMI e também apresentou uma redução na duração da sedação. No entanto, seus autores não incluíram avaliações musculares no ensaio clínico ${ }^{18}$. Da mesma forma, a EENM foi associada ao aumento da força do músculo estimulado em pacientes sépticos e pode ser uma forma de prevenção da fraqueza muscular nessa população ${ }^{10}$. Um fato interessante foi que a melhora foi observada principalmente em pacientes mais graves e mais fracos ${ }^{10}$. Então, a melhor recuperação pode ser influenciada pelo estado clínico dos pacientes em que aqueles mais gravemente enfermos perdem mais massa muscular e podem se beneficiar mais da intervenção ${ }^{24}$. No entanto, a EENM não influenciou a espessura da camada muscular, mas foi associada a uma maior recuperação da força muscular durante a permanência na UTI'12.

Quanto ao tempo necessário para o EENM produzir efeitos sobre os músculos e guiar o protocolo, Silva et al. 
(2019) demonstraram ser necessário, pelo menos, sete dias para prevenir distúrbios de arquitetura muscular e tratar fraqueza e quatorze dias para tratar desordem eletrofisiológica neuromuscular (DEN) induzida pela neuromiopatia. Sua intervenção em pacientes com Traumatismo Crânio Encefálico (TCE) reduziu a atrofia muscular, a incidência de DEN e a fraqueza muscular, medidas por ultrassom, eletrofisiologia neuromuscular e pico de força evocado ${ }^{20}$. Protocolos de EENM devem avaliar a excitabilidade neuromuscular através do teste de eletrodiagnóstico de estímulo em pacientes com distúrbios eletrofisiológicos neuromusculares para aplicar estímulos elétricos capazes de gerar torque significativo e melhorar a resposta muscular contrátil ${ }^{49}$.

Quanto ao modo de aplicação da corrente elétrica, Nakamura et al. (2019) utilizaram eletrodo tipo cinto que substitui os tradicionais eletrodos descartáveis e induziram contrações simultâneas do músculo esquelético de toda a parte inferior do corpo. Concluíram que a estimulação tem potencial de inibir a perda de volume muscular em cuidados intensivos na fase aguda. No entanto, outros desfechos como tempo de ventilação mecânica, de UTI e do hospital não foram estatisticamente significativos ${ }^{22}$.

Estudo de revisão mostrou ser uma intervenção segura, viável, benéfica e bem tolerada com poucos eventos adversos, sem necessidade de interromper a terapia. No entanto, os parâmetros utilizados devem ser baseados em critérios de evidência e aplicados por profissionais treinados ${ }^{48}$. Um ponto positivo da EENM é que não requer cooperação do paciente, então pode ser facilmente aplicada imediatamente após a admissão do paciente na UTI50.

Quanto ao modo de medição da força e espessura muscular, a maioria avaliou a força manualmente pelo Medical Research Council (MRC). Esta escala de cinco pontos, no entanto, pode produzir resultados diferentes inter até intraindividualmente em diferentes momentos ${ }^{11}$. Permanece uma medida subjetiva, requer colaboração do sujeito e o nível de consciência e a presença de delirium também podem afetar essas medidas; no entanto, do ponto de vista clínico, o MRC é válido e significativo. Por outro lado, em pacientes gravemente fracos, as medidas de força usando um dinamômetro podem ser difíceis de realizar ${ }^{10}$. A avaliação de alterações na massa muscular com tomografia computadorizada ou ultrassonografia proporcionará resultados objetivos ${ }^{11}$. No entanto, a ultrassonografia pode sofrer influência do edema e gordura e pode não captar a região em que ocorreu o efeito ${ }^{22}$.

As frequências variaram de 20 a 100 Hertz e a largura do pulso de 300 a 500 us e a intensidade capaz de produzir contração visível ou evocar contrações máximas. O tempo de aplicação também variou de 30 a 60 min., uma ou duas vezes ao dia. Alguns utilizaram os pontos motores. Isso demonstra a aplicação de protocolos variados, sem uma padronização entre os estudos, dificultando comparações. Não se sabe, por exemplo, se um período mais longo em dias de aplicação pode ter um impacto diferente. Não está claro nos estudos se houve contração muscular adequada durante a aplicação e muitos investigaram somente as extremidades inferiores ou apenas o quadríceps femoral. A falta de resposta contrátil pode ser uma das causas de resultados contraditórios. O estudo com diferentes grupos musculares e diferentes pacientes apresentaram uma resposta variável ligada a múltiplos fatores do desenvolvimento da FMA-UTI?.

Enfim, os protocolos da EENM ainda não elucidam completamente a dose ideal, variação na intensidade, na duração, nas repetições e no local de aplicação ${ }^{20}$. Praticamente não são registrados eventos adversos e quando ocorrem não impedem a realização do exercício ${ }^{48}$. Os estudos foram heterogêneos com protocolos, parâmetros para eletroestimulação, tamanhos amostrais e desfechos diferentes dificultando as comparações. Novos estudos são necessários, principalmente para estabelecer um consenso quanto à modulação adequada.

\section{Limitações gerais dos estudos}

Quanto as limitações gerais de todos os estudos, pode-se citar a falta de alocação oculta que levou a um viés de seleção, não proporcionando a mesma chance do participante pertencer ao grupo e a falta de cegamento nas diversas partes do estudo. Em algumas intervenções em fisioterapia é difícil realizar cegamento principalmente de quem administra a terapia ou do participante, mas as avaliações dos resultados, na sua maioria, foram feitas de maneira cega. Outra limitação foi o tamanho das amostras que, quando muito pequena, prejudica a análise dos resultados, comprometem a validade interna dos estudos com estimativas imprecisas. A divisão em categorias norteadoras pode gerar alguma confusão já que a EENM e o exercício em cicloergômetro também fazem parte a mobilização precoce. A separação somente ocorreu para fins didáticos e para facilitar a discussão. No entanto, a heterogeneidade dos resultados ocorreu tanto devido as diferentes intervenções como também com as mesmas intervenções pertencentes a mesma categoria norteadora.

\section{CONCLUSÃO}

No âmbito da intervenção fisioterapêutica motora, nos últimos anos, o interesse tem se concentrado na mobilização precoce, estimulação elétrica neuromuscular e utilização do cicloergômetro para realização de exercícios. No entanto, são necessárias conclusões mais definitivas e intervenções mais padronizadas, já que os desfechos foram heterogêneos e os estudos analisados foram de média qualidade metodológica. Considera-se, nesse sentido, que as evidências ainda são insuficientes para guiar de uma maneira clara a prática clínica. Pesquisas futuras devem abordar as lacunas relacionadas à seleção dos pacientes, dosagem da reabilitação e o início da terapia nos pacientes de cuidados críticos. 
FONTE DE FINANCIAMENTO

Nada a declarar

\section{CONFLITO DE INTERESSE}

Nada a declarar

\section{REFERÊNCIAS}

1. Machado AS, Pires-Neto RC, Carvalho MTX, Soares JC, Cardoso DM, Alburquerque IM. Efeito do exercício passivo em cicloergômetro na força muscular, tempo de ventilação mecânica e internação hospitalar em pacientes críticos: ensaio clínico randomizado. J Bras Pneumol. 2017;43(2):1349. http://dx.doi.org/10.1590/s1806-37562016000000170. PMid:28538781.

2. Wright SE, Thomas K, Watson G, Baker C, Bryant A, Chadwick TJ, et al. Intensive versus standard physical rehabilitation therapy in the critically ill (EPICC): A multicentre, parallelgroup, randomised controlled trial. Thorax. 2018;73(3):21321. http://dx.doi.org/10.1136/thoraxjnl-2016-209858. PMid:28780504.

3. Moss M, Nordon-Craft A, Malone D, Van Pelt D, Frankel SK, Warner ML, et al. A randomized trial of an intensive physical therapy program for patients with acute respiratory failure. Am J Respir Crit Care Med. 2016;193(10):1101-10. http:// dx.doi.org/10.1164/rccm.201505-10390C. PMid:26651376.

4. Yosef-Brauner O, Adi N, Ben Shahar T, Yehezkel E, Carmeli E. Effect of physical therapy on muscle strength, respiratory muscles and functional parameters in patients with intensive care unit acquired weakness. Clin Respir J. 2015;9(1):1-6. http://dx.doi.org/10.1111/crj.12091. PMid:24345055.

5. Eggmann S, Verra ML, Luder G, Takala J, Jakob SM. Effects of early, combined endurance and resistance training in mechanically ventilated, critically ill patients: A randomised controlled trial. PLoS One. 2018;13(11):e0207428. http:// dx.doi.org/10.1371/journal.pone.0207428. PMid:30427933.

6. Santos LMH, Novaes APL, Dantas FMNA, Ribeiro LC, Castro $\mathrm{CMMB}$, França EET. Acute effect of passive cycloergometry on the cardiovascular system and respiratory mechanics of critically ill patients: a randomized controlled trial. Fisioter Mov. 2019;32:e003232. http://dx.doi.org/10.1590/19805918.032.ao32.

7. Grunow Jj, Goll M, Carbon NM, Liebl ME, Weber-Carstens S, Wollersheim T. Differential contractile response of critically ill patients to neuromuscular electrical stimulation. Crit Care. 2019;23(1):1-12. http://dx.doi.org/10.1186/s13054-019-2540-4.

8. Almeida TSO, Almeida TSO, Ramalho SNL. Distúrbios musculoesqueléticos em idosos: uma avaliação da qualidade de vida após exercícios aquáticos. Revista InterScientia. 2017;5(2):51-68.

9. Forte ECN, Pires DEP, Trigo SVV, Martins MMFPS. A hermenêutica e o software atlas.ti: união promissora. Texto Contexto Enferm. 2017;26(4):1-8. http://dx.doi. org/10.1590/0104-07072017000350017.

10. Rodriguez PO, Setten M, Maskin LP, Bonelli I, Vidomlansky SR, Attie S. et al. Muscle weakness in septic patients requiring mechanical ventilation: protective effect of transcutaneous neuromuscular electrical stimulation. J Criti Care. 2012;27(3):319.

11. Akar O, Günay E, Sarinc Ulasli S, Ulasli AM, Kacar E, Sariaydin $M$, et al. Efficacy of neuromuscular electrical stimulation in patients with COPD followed in intensive care unit. Clin
Respir J. 2017;11(6):743-50. http://dx.doi.org/10.1111/ crj.12411. PMid:26597394.

12. Fischer A, Spiegl M, Altmann K, Winkler A, Salamon $A$, Themessl-Huber $M$, et al. Muscle mass, strength and functional outcomes in critically ill patients after cardiothoracic surgery: does neuromuscular electrical stimulation help? The Catastim 2 randomized controlled trial. Crit Care. 2016;20(1):30. http://dx.doi.org/10.1186/ s13054-016-1199-3. PMid:26825278.

13. Morris PE, Berry MJ, Files DC, Thompson JC, Hauser J, Flores $L$, et al. Standardized rehabilitation and hospital length of stay among patients with acute respiratory failure: a randomized clinical trial. JAMA. 2016;315(24):2694-702. http://dx.doi.org/10.1001/jama.2016.7201. PMid:27367766.

14. Schaller SJ, Anstey M, Blobner M, Edrich T, Grabitz SD, Gradwohl-Matis I, et al. Early, goal-directed mobilisation in the surgical intensive care unit: a randomised controlled trial. Lancet. 2016;388(10052):1377-88. http://dx.doi. org/10.1016/S0140-6736(16)31637-3. PMid:27707496.

15. Fossat G, Baudin F, Courtes L, Bobet S, Dupont A, Bretagnol $A$, et al. Effect of In-Bed leg cycling and electrical stimulation of the quadriceps on global muscle strength in critically ill adults: a randomized clinical trial. JAMA. 2018;320(4):368-78. http://dx.doi.org/10.1001/jama.2018.9592. PMid:30043066.

16. Hickmann CE, Castanares-Zapatero D, Deldicque L, Van Den Bergh P, Caty G, Robert A, et al. Impact of very early physical therapy during septic shock on skeletal muscle: a randomized controlled trial. Crit Care Med. 2018;46(9):1436-43. http://dx.doi. org/10.1097/CCM.0000000000003263. PMid:29957714.

17. Medrinal C, Combret Y, Prieur G, Quesada AR, Bonnevie T, Gravier E, et al. Comparison of exercise intensity during four early rehabilitation techniques in sedated and ventilated patients in ICU: a randomised cross-over trial. Crit Care. 2018;22(1):110. http://dx.doi.org/10.1186/s13054-018-20300. PMid:29703223.

18. Santos FV, Cipriano JRG, Vieira L, Güntze L, Chiappa AM, Cipriano GBF, et al. Neuromuscular electrical stimulation combined with exercise decreases duration of mechanical ventilation in ICU patients: A randomized controlled trial. Physiother Theory Pract. 2018;36(05):580-8. http://dx.doi. org/10.1080/09593985.2018.1490363. PMid:30321084.

19. Sarfati C, Moore A, Pilorge C, Amaru P, Mendialdua P, Rodet $E$, et al. Efficacy of early passive tilting in minimizing ICUacquired weakness: a randomized controlled trial.J Crit Care. 2018;46:37-43. http://dx.doi.org/10.1016/j.jcrc.2018.03.031. PMid:29660670.

20. Silva PE, Marqueti RC, Carvalho KL, Araujo AET, Castro J, Silva VM, et al. Neuromuscular electrical stimulation in critically ill traumatic brain injury patients attenuates muscle atrophy, neurophysiological disorders, and weakness: a randomized controlled trial. J Intensive Care. 2019;7(1):1-13. http://dx.doi. org/10.1186/s40560-019-0417-x. PMid:31890221.

21. Chen YH, Hsiao HF, Li LF, Chen NH, Huang CC. Effects of electrical muscle stimulation in subjects undergoing prolonged mechanical ventilation. Respir Care. 2019;64(3):262-71. http://dx.doi.org/10.4187/ respcare.05921. PMid:30723168.

22. Nakamura K, Kihata A, Naraba H, Kanda N, Takahashi $Y$, Sonoo T, et al. Efficacy of belt electrode skeletal muscle electrical stimulation on reducing the rate of muscle volume loss in critically ill patients: A randomized controlled trial. J Rehabil Med. 2019;51(9):705-11. http://dx.doi. org/10.2340/16501977-2594. PMid:31544949.

23. Schujmann DS, Teixeira Gomes T, Lunardi AC, Zoccoler Lamano $M$, Fragoso A, Pimentel $M$, et al. Impact of a progressive 
mobility program on the functional status, respiratory, and muscular systems of ICU patients: a randomized and controlled trial. Crit Care Med. 2020;48(4):491-7. http://dx.doi. org/10.1097/CCM.0000000000004181. PMid:32205595.

24. Segers J, Vanhorebeek I, Langer D, Charususin N, Wei W, Frickx $B$, et al. Early neuromuscular electrical stimulation reduces the loss of muscle mass in critically ill patients - A within subject randomized controlled trial.J Crit Care. 2021;62:65-71. http://dx.doi.org/10.1016/j.jcrc.2020.11.018. PMid:33285371.

25. Nedel WL, Silveira F. Os diferentes delineamentos de pesquisa e suas particularidades na terapia intensiva. Rev Bras Ter Intensiva. 2016;28(3):256-60. PMid:27737421.

26. Silva AR, Miranda HAAA, Miranda DB, Benito LAO, Costa CMA, Souza SESM, BarrosJF. Análise da prática clínica fisioterapêutica baseada em evidências. Revista Digital. 2016; 21:219.

27. Tipping CJ, Harrold M, Holland A, Romero L, Nisbet $T$, Hodgson C. The effects of active mobilisation and rehabilitation in ICU on mortality and function: a systematic review. Intensive Care Med. 2017;43(2):171-83. http://dx.doi. org/10.1007/s00134-016-4612-0. PMid:27864615.

28. Vorona S, Sabatini U, Al-Maqbali S, Bertoni M, Dres M, Bissett B, et al. Inspiratory muscle rehabilitation in critically ill adults. A systematic review and meta-analysis. Ann Am Thorac Soc. 2018;15(6):735-44. http://dx.doi.org/10.1513/ AnnalsATS.201712-9610C. PMid:29584447.

29. McWilliams D, Jones C, Atkins G, Hodson J, Whitehouse $\mathrm{T}$, Veenith $\mathrm{T}$, et al. Earlier and enhanced rehabilitation of mechanically ventilated patients in critical care: a feasibility randomised controlled trial.J Crit Care. 2018;44:407-12. http:// dx.doi.org/10.1016/j.jcrc.2018.01.001. PMid:29331668.

30. Cameron S, Ball I, Cepinskas G, Choong K, Doherty TJ, Ellis CG, et al. Early mobilization in the critical care unit: A review of adult and pediatric literature. J Crit Care. 2015;30(4):664-72. http://dx.doi.org/10.1016/j. jcrc.2015.03.032. PMid:25987293.

31. Hickmann CE, Castanares-Zapatero D, Bialais E, Dugernier J, Tordeur A, Colmant L, et al. Teamwork enables high level of early mobilization in critically ill patients. Ann Intensive Care. 2016;6(1):80. http://dx.doi.org/10.1186/s13613-016-0184-y. PMid:27553652.

32. Ding N, Zhang Z, Zhang C, Yao L, Yang L, Jiang B, et al. What is the optimum time for initiation of early mobilization in mechanically ventilated patients? A network meta-analysis. PLoS One. 2019;14(10):e0223151. http://dx.doi.org/10.1371/ journal.pone.0223151. PMid:31589642.

33. Pinto BF, Pinto BF, Dias EHF. Efeitos sistêmicos da mobilização precoce em pacientes adultos internados na unidade de terapia intensiva: revisão atualizada. Fisioter Bra. 2019;19(6):857-65. http://dx.doi.org/10.33233/fb.v19i6.2118.

34. Arias-Fernández P, Romero-Martin M, Gómez-Salgado J, Fernández-García D. Rehabilitation and early mobilization in the critical patient: systematic review. J Phys Ther Sci. 2018;30(9):1193-201. http://dx.doi.org/10.1589/ jpts.30.1193. PMid:30214124.

35. Zhang L, Hu W, Cai Z, Liu J, Wu J, Deng Y, et al. Early mobilization of critically ill patients in the intensive care unit: A systematic review and meta-analysis. PLoS One. 2019;14(10):e0223185. http://dx.doi.org/10.1371/journal. pone.0223185. PMid:31581205.

36. Aquim EE, Bernardo WM, Buzzini RF, Azeredo NSG, Cunha LS, Damasceno MCP, et al. Diretrizes Brasileiras de Mobilização Precoce em Unidade de Terapia Intensiva. Ver Bras Ter Intensiva. 2019;31(4):434-43.

37. Sommers J, Engelbert RH, Dettling-Ihnenfeldt D, Gosselink $\mathrm{R}$, Spronk PE, Nollet F, et al. Physiotherapy in the intensive care unit: an evidence-based, expert driven, practical statement and rehabilitation recommendations. Clin Rehabil. 2015;29(11):1051-63. http://dx.doi. org/10.1177/0269215514567156. PMid:25681407.

38. McWilliams D, Weblin J, Atkins G, Bion J, Williams J, Elliott $C$, et al. Enhancing rehabilitation of mechanically ventilated patients in the intensive care unit: a quality improvement project. J Crit Care. 2015;30(1):13-8. http:// dx.doi.org/10.1016/j.jcrc.2014.09.018. PMid:25316527.

39. Wahab R, Yip NH, Chandra S, Nguyen M, Pavlovich KH, Benson $\mathrm{T}$, et al. The implementation of an early rehabilitation program is associated with reduced length of stay: a multiICU study. J Intensive Care Soc. 2016;17(1):2-11. http:// dx.doi.org/10.1177/1751143715605118. PMid:28979452.

40. Fontela PC, Forgiarini LA Jr, Friedman G. Clinical attitudes and perceived barriers to early mobilization of critically ill patients in adult intensive care units. Rev Bras Ter Intensiva. 2018;30(2):187-94. http://dx.doi.org/10.5935/0103507X.20180037. PMid:29995084.

41. Kho ME, Martin RA, Toonstra AL, Zanni JM, Mantheiy EC, Nelliot $A$, et al. Feasibility and safety of in-bed cycling for physical rehabilitation in the intensive care unit. J Crit Care. 2015;30(6):1419. http://dx.doi.org/10.1016/j. jcrc.2015.07.025. PMid:26318234.

42. Hashem MD, Parker AM, Needham DM. Early mobilization and rehabilitation of patients who are critically ill. Chest. 2016;150(3):722-31. http://dx.doi.org/10.1016/j. chest.2016.03.003. PMid:26997241.

43. Dafoe S, Chapman MJ, Edwards S, Stiller K. Overcoming barriers to the mobilisation of patients in an intensive care unit. Anaesth Intensive Care. 2015;43(6):719-27. http:// dx.doi.org/10.1177/0310057X1504300609. PMid:26603796.

44. Barber EA, Everard T, Holland AE, Tipping C, Bradley SJ, Hodgson CL. Barriers and facilitators to early mobilisation in Intensive Care: A qualitative study. Aust Crit Care. 2015;28(4):177-82. http://dx.doi.org/10.1016/j. aucc.2014.11.001. PMid:25533868.

45. Coutinho WM, Santos LJ, Fernandes J, Vieira SRR, Forgiarini Júnior LA, Dias AS. Efeito agudo da utilização do cicloergômetro durante atendimento fisioterapêutico em pacientes críticos ventilados mecanicamente. Fisioter Pesqui. 2016;23(3):27883. http://dx.doi.org/10.1590/1809-2950/15549123032016.

46. Kimawi I, Lamberjack B, Nelliot A, Toonstra L, Zanni J, Huang $M$, et al. Safety and feasibility of a protocolized approach to in-bed cycling exercise in the intensive care unit: quality improvement project. Phys Ther. 2017;97(6):593-02. http:// dx.doi.org/10.1093/ptj/pzx034. PMid:28379571.

47. Nickels MR, Aitken LM, Barnett AG, Walsham J, Mcphail SM. Acceptability, safety, and feasibility of in-bed cycling with critically ill patients. Aust Crit Care. 2020;33(3):236-43. http:// dx.doi.org/10.1016/j.aucc.2020.02.007. PMid:32317212.

48. Sachetti A, Carpes MF, Dias AS, Sbruzzi G. Segurança no uso da eletroestimulação neuromuscular em pacientes graves: revisão sistemática. Rev Bras Ter Intensiva. 2018;30(2):21925. PMid:29995088.

49. Silva PE, Babault N, Mazullo JB, de Oliveira TP, Lemos BL, Carvalho VO, et al. Safety and feasibility of a neuromuscular electrical stimulation chronaxie-based protocol in critical ill patients: A prospective observational study. J Crit Care. 2017;37:141-8. http://dx.doi.org/10.1016/j.jcrc.2016.09.012. PMid:27732921.

50. Leite MA, Osaku EF, Albert J, Costa CRLM, Garcia AM, Czapiesvski FN, et al. Effects of neuromuscular electrical stimulation of the quadriceps and diaphragm in critically III patients: a pilot study. Crit Care Res Pract. 2018;2018:4298583. http://dx.doi.org/10.1155/2018/4298583. PMid:30123586. 